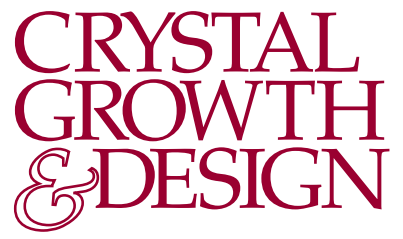

\title{
Hydrogen Bonding Guests Direct the Packing of a Small Organic Cage Molecule
}

\author{
Thomas Anglim Lagones, Stephanie A. Boer, and Nicholas G. White*(i) \\ Research School of Chemistry, The Australian National University, Canberra, Australian Capital Territory, Australia
}

Supporting Information

ABSTRACT: A small organic cage molecule (1) containing six nitrile groups was crystallized in the presence of a number of guests with hydrogen bond donor groups, and from different solvents. In total, eight crystal structures of 1 were obtained, six of which are guest-free and two of which are cocrystals. When the guest was resorcinol or pyrogallol, cocrystals did not form, but the presence of the guests directed formation of new crystalline phases that were not observed when the cage was crystallized alone. When the guest was hydroquinone or diaminobenzene, it was possible to isolate cocrystals where the guest hydrogen bonds to some of the nitrile groups of the cage.

\section{INTRODUCTION}

Molecular cages are a class of molecules containing a pore in their center, and numerous examples of both organic ${ }^{1-3}$ and metal-organic cages $^{4-8}$ have been reported. While by definition cages contain a void (i.e., are usually intrinsically porous), they are often nonporous as solids, because the cages pack in such a way that the pores present in the material do not align. Nonetheless, cages are attractive candidates for porous materials as they are solution processable and can often be prepared in a modular fashion. ${ }^{3}$

To benefit from the advantages of cages while overcoming their tendency to nonporosity in the solid state, there is considerable interest in developing methods to control the organization of cages in three-dimensional (3D) solids. Ideally, this would involve taking a discrete cage molecule and using additional interactions to drive formation of a porous crystalline 3D network. Significant progress has recently been made in this area with covalent bond formation, 9,10 metalligand coordination, ${ }^{1-20}$ and noncovalent forces ${ }^{21-28}$ being used to drive framework assembly.

An alternative approach would be to cocrystallize appropriately functionalized cage molecules with hydrogen bonding guests and use these guests to direct the formation of 3D superstructures (i.e., hydrogen bonded frameworks ${ }^{29-31}$ ). In this manner, one cage could potentially be used to assemble several cage networks simply by using a variety of guest molecules. Presumably, relatively strong hydrogen bonding groups would be needed to prevent collapse of the framework.

As a first step toward hydrogen bonded cage networks, we report our investigation into the crystallization of cage 1 in the presence of various hydrogen bonding guests (hydroquinone, HQ resorcinol, Res; pyrogallol, PG; 1,3-diaminobenzene, DAB; Figure 1). Compound 1, first reported by Katz, ${ }^{32}$ contains six potentially hydrogen bond-accepting nitrile groups that could interact with hydrogen bond-donating guest

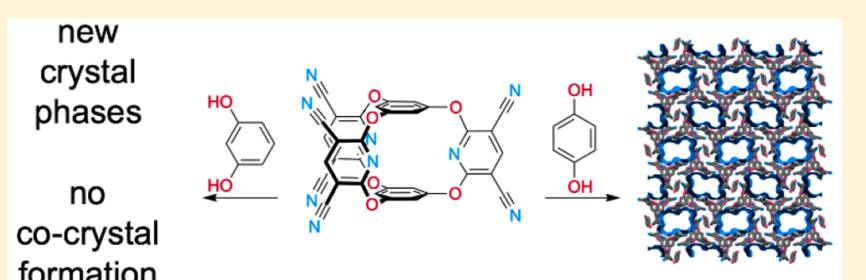

formation
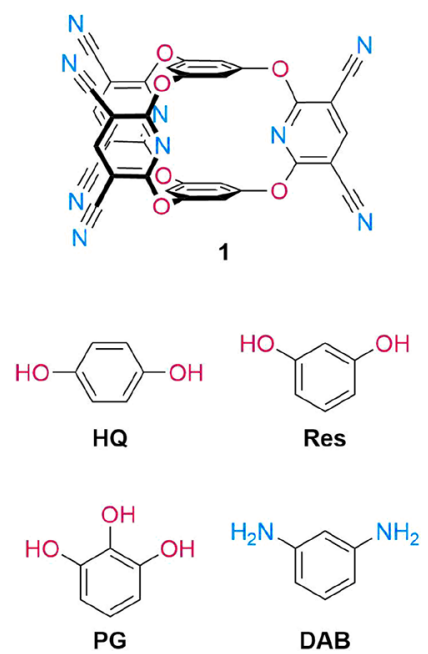

Figure 1. Structure of organic cage molecule $\mathbf{1}$ and potential hydrogen bonding guests used in this study.

molecules. These nitrile groups are not particularly strong hydrogen bond acceptors ${ }^{33,34}$ and so are unlikely to lead to stable hydrogen bonded networks; ${ }^{35}$ however, this cage is easy to synthesize, allowing for a detailed study of its crystallization behavior. We demonstrate that 1 possesses a rich crystallization landscape and that hydrogen bonding guests can be used to control crystallization, ${ }^{36}$ with or without the formation of cocrystals. ${ }^{37-39}$

Received: April 29, 2019

Revised: $\quad$ May 31, 2019

Published: June 18, 2019 

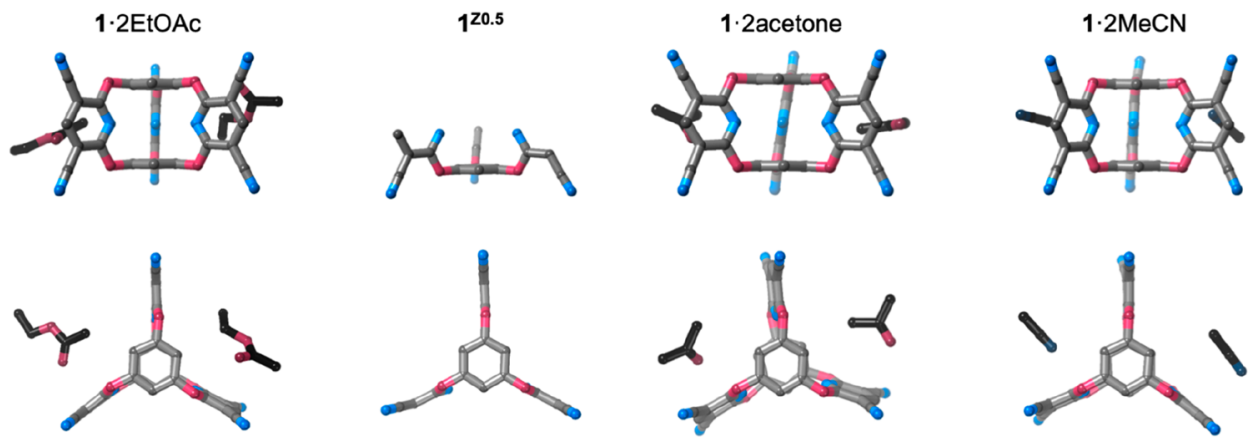

Figure 2. Views of the asymmetric units of different solvatomorphs of 1 (hydrogen atoms omitted for clarity). PLATON-SQUEEZE ${ }^{41}$ was used to include ill-defined disordered solvent in the structure of $1^{\mathrm{Z0.5}}$; the structure of $1.2 \mathrm{EtOAc}$ was reported by Katz et al. ${ }^{32}$

\section{RESULTS AND DISCUSSION}

Solvatomorphs of 1 . Before studying the interaction of 1 with hydrogen bonding guest molecules, we investigated how crystallization solvent affected crystal packing. ${ }^{40}$ Katz has reported the crystal structure of $1 \cdot 2 \mathrm{EtOAc}$, which was prepared by crystallization from hot ethyl acetate; ${ }^{32}$ this structure has $Z^{\prime}$ $=1$. We crystallized 1 by vapor diffusion of diethyl ether into an ethyl acetate solution of the cage, which gave a different crystal form, $1^{\mathrm{Z0} .5}$ with one-half of the cage in the asymmetric unit. Two regions of ill-defined electron density were present about a special position; these could not be sensibly modeled, and so PLATON-SQUEEZE ${ }^{41}$ was used to include the electron density in the refinement. Crystallization of $\mathbf{1}$ by vapor diffusion of diethyl ether into acetone or acetonitrile solutions gave the solvatomorphs $1 \cdot 2$ acetone and $1 \cdot 2 \mathrm{MeCN}$.

The structures of these four solvatomorphs are shown in Figure 2: generally, the structures are very similar with two solvents per cage molecule located between the pairs of dicyanopyridine "wings" of the cage. In the case of $1^{\mathrm{Z0.5}}$, the regions of solvent density are of similar size and in similar locations to the other three solvatomorphs, suggesting a similar solvent content. While the structures appear quite similar, subtle differences in packing are apparent (Figures S16-S23) with the larger solvent molecules forcing the cages slightly further apart. These small changes caused by solvent are a notable contrast to those caused by other hydrogen bonding guests (see later).

Interaction of 1 with Guests in Solution. Having observed that solvent had only a subtle effect on the crystallization of $\mathbf{1}$, we next investigated the effect of hydrogen bond donors on crystallization. We initially studied whether 1 could interact with hydrogen bonding guests in solution. The cage is soluble in a range of moderately polar to polar solvents (e.g., EtOAc, acetone, $\mathrm{MeCN}$ ) but not in chlorinated solvents. Addition of guests to $\mathbf{1}$ in acetone- $d_{6}$ showed only very minor shifts in the ${ }^{1} \mathrm{H}$ NMR spectra indicating very weak interactions in this solvent (Figures S13-S15).

Quantitative NMR binding studies were undertaken using HQ and PG in this solvent, but in both cases, 1:1 association constants were calculated to be $\ll 1 \mathrm{M}^{-1}$ (see Supporting Information for more details). Attempts to determine association constants in less polar solvents were thwarted because either 1 is not soluble in these solvents $\left(\mathrm{CD}_{2} \mathrm{Cl}_{2}\right.$, $\left.\mathrm{CDCl}_{3}, \mathrm{THF}-d_{8}\right)$ or the solvents are prohibitively expensive (EtOAc- $\left.d_{8}\right)$.

Effect of Guests on Solid State Structures. While negligible interactions are present between $\mathbf{1}$ and guests in acetone- $d_{6}$ solution, we were still interested in the effect of these guests on the crystallization behavior of $\mathbf{1}$. To study this, we obtained crystals by diffusing diethyl ether vapor into an EtOAc solution of the cage and guests.

1 and Res. Crystallization of 1 in the presence of one molar equivalent of Res gave colorless block-like crystals. X-ray crystallography and ${ }^{1} \mathrm{H}$ NMR spectroscopy showed that no Res was incorporated in the structure, but interestingly the crystals are not the same as those when the cage is crystallized under the same conditions in the absence of guest. In the absence of guest, $\mathbf{1}^{\mathbf{Z 0 . 5}}$ is formed; when Res is present during crystallization, a new phase is formed which has $Z^{\prime}=2$. This phase, $1^{\mathrm{Z} 2}$, has one diethyl ether molecule present per molecule of cage, i.e., two in the asymmetric unit (Figure 3 ). When 2 or 6 equiv of Res was used, the same crystal phase was produced, along with a small amount of amorphous material.

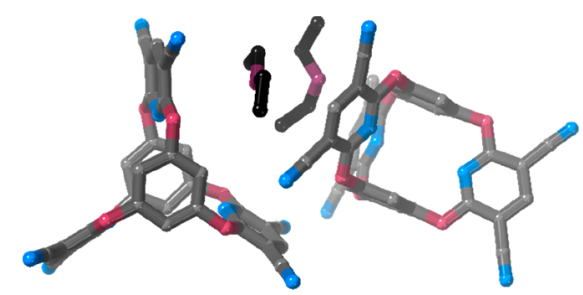

Figure 3. Asymmetric unit of $\mathbf{1}^{\mathrm{Z} 2}$ phase (hydrogen atoms omitted for clarity).

1 and PG. Crystallization of 1 in the presence of 1 equiv of PG gave two different types of crystals. The major product were prisms, while a small amount of block-shaped crystals was also formed. X-ray crystallography and ${ }^{1} \mathrm{H}$ NMR spectroscopy revealed that neither type of crystal contained the guest. X-ray crystallography showed that the minor block product was $\mathbf{1}^{\mathrm{Z2}}$, while the major product was a new phase $1^{\mathrm{Z3}}$, with $Z^{\prime}=3$ (Figure 4). The asymmetric unit of $\mathbf{1}^{\mathrm{Z3}}$ contains one diethyl ether solvent molecule as well as large regions that appear to contain disordered/partial occupancy diethyl ether and ethyl acetate solvent molecules. These could not be modeled sensibly, and so PLATON-SQUEEZE ${ }^{41}$ was used to include the electron density in the model. When 2 or 6 equiv of PG was used, crystals of $1^{\mathrm{Z} 3}$ were the only product that formed.

1 and $H Q$. Crystallization of 1 in the presence of 1 equiv of HQ gave crystals of $\mathbf{1}^{\mathrm{Z} 2}$; when 2 equiv of HQ was used, one or two crystals of $1^{\mathrm{Z} 2}$ formed, but the major product was the cocrystal $\mathbf{1} \cdot(\mathrm{HQ})_{0.5}$ (as determined by NMR spectroscopy and 


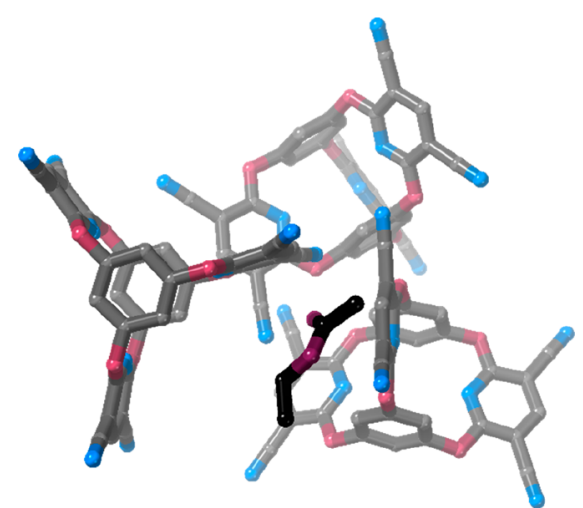

Figure 4. Asymmetric unit of $1^{\mathrm{Z} 3}$ phase (hydrogen atoms omitted for clarity; PLATON-SQUEEZE ${ }^{41}$ used).

visual analysis of crystals, see Figures S3 and S7). Using 6 equiv of HQ gave exclusively $\mathbf{1} \cdot(\mathbf{H Q})_{0.5}$ in very good yield (95\%).

1 and $D A B$. Adding an ethyl acetate solution of 1 to $D A B$ did not result in a clear solution (as was the case when PG, Res, and HQ were used). When an ethyl acetate solution of 1 was carefully layered with an ethyl acetate solution of DAB, single crystals of $\mathbf{1} \cdot(\mathrm{DAB})_{2}$ formed. If only 1 equiv of $\mathrm{DAB}$ was used, these crystals formed in low yield (15\%), while using 2 or 6 equivalents gave better yields ( 45 and $65 \%$, respectively).

Cocrystals. The asymmetric unit of $\mathbf{1}(\mathrm{HQ})_{0.5}$ contains one molecule of the cage and half a molecule of HQ as well as a region of diffuse electron density, which appears to result from disordered solvent molecules. This electron density could not be refined sensibly, and so PLATON-SQUEEZE ${ }^{41}$ was used to include the density in the refinement. The hydroquinone $\mathrm{O}-\mathrm{H}$ group forms a relatively short hydrogen bond to a nitrile moiety from the cage $(\mathrm{H} \cdots \mathrm{N}=1.98 \AA$, $69 \%$ of the sum of the van der Waals radii ${ }^{42}$ of $\mathrm{H}$ and $\mathrm{N}$ ), with crystallographic symmetry, meaning that this HQ group links two cages together in the solid state (Figure 5). Relatively short stacking interactions between the electron-rich $\mathrm{HQ}$ ring and electrondeficient dicyanopyridine groups from the cage further direct the crystal packing (centroid $\cdots$ centroid distances: $3.53 \AA$ ) .

Interestingly, this combination of noncovalent interactions leads to a relatively open structure containing large channels (Figure 6). These channels are apparently filled with disordered solvent molecules, and these voids make up 29\% of the unit cell volume. ${ }^{43}$

The asymmetric unit of $\mathbf{1} \cdot(\mathbf{D A B})_{2}$ contains half a molecule of 1 and a complete molecule of DAB. Interestingly, this structure does not contain any solvent molecules (Figure 7), but instead crystallizes in a manner similar to $1 \cdot 2 \mathrm{EtOAc}, \mathbf{1}^{\mathrm{Z0.5}}$, $1 \cdot 2$ acetone, and $1.2 \mathrm{MeCN}$, with the $\mathbf{D A B}$ guests filling the spaces occupied by solvent molecules in those structures. One of the DAB $\mathrm{NH}_{2}$ groups forms moderate length hydrogen bonds with nitrile groups of $1(\mathrm{H} \cdots \mathrm{N}=2.21,2.27 \AA$; 77, 79\% of the sum of the van der Waal radii ${ }^{42}$ of $\mathrm{H}$ and $\mathrm{N}$ ) such that a 1D H-bonded polymeric structure is formed. The other $\mathrm{NH}_{2}$ does not take part in any short hydrogen bonds ( $\mathrm{H}$-.acceptor $>2.5 \AA$ ), and no short stacking interactions are observed either.

\section{DISCUSSION}

The effect of the hydrogen bonding guests on the solid state structure of $\mathbf{1}$ is summarized in Table 1 . It is clear that the presence of hydrogen bonding guests has a significant
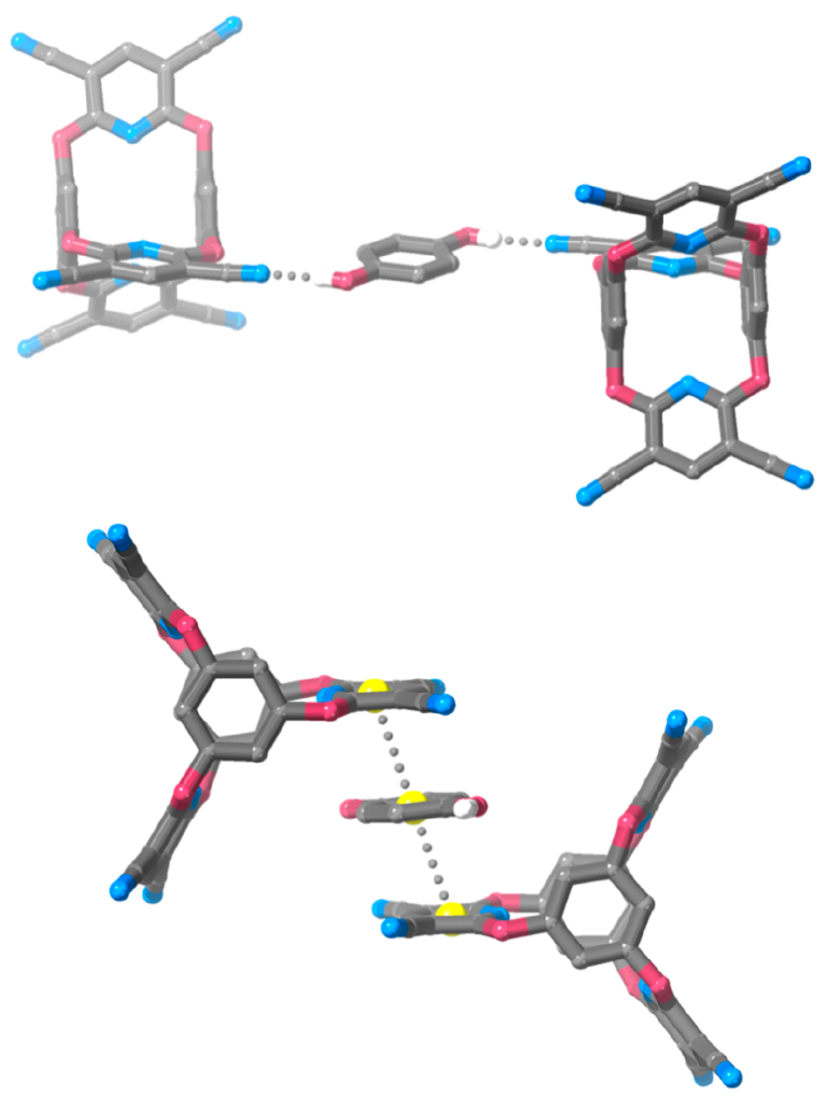

Figure 5. View of the noncovalent interactions in the crystal structure of $1 \cdot(\mathrm{HQ})_{0.5}$ (most hydrogen atoms omitted for clarity, yellow spheres represent centroids of aromatic rings).

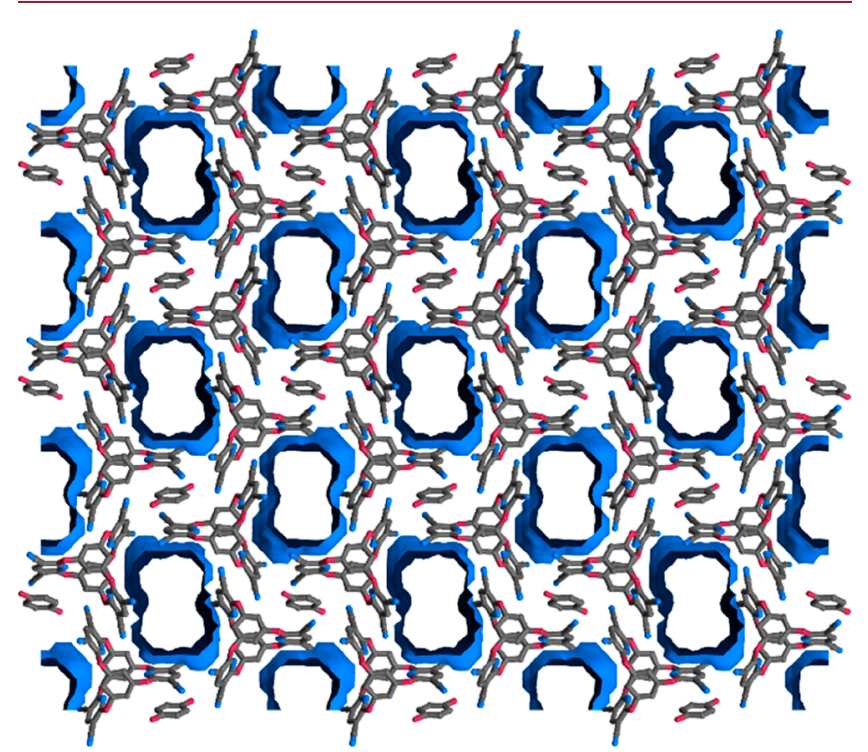

Figure 6. View of large channels running through the structure of $\mathbf{1}$. $(\mathbf{H Q})_{0.5}$. Blue channels calculated using Mercury ${ }^{44}$ with a probe radius of $1.82 \AA$.

structural impact on $\mathbf{1}$, even when the guest is not included in the crystal, i.e., when a cocrystal does not form. When PG is the guest, ${ }^{45}$ it is possible to isolate $1^{\mathrm{Z} 3}$ when a small amount of guest is used and $\mathbf{1}^{\mathrm{Z2}}$ with a greater amount of guest. In the case of $H Q$ a small amount of guest gives $\mathbf{1}^{\mathrm{Z} 2}$, while adding a larger amount of guest leads to the formation of cocrystals. 

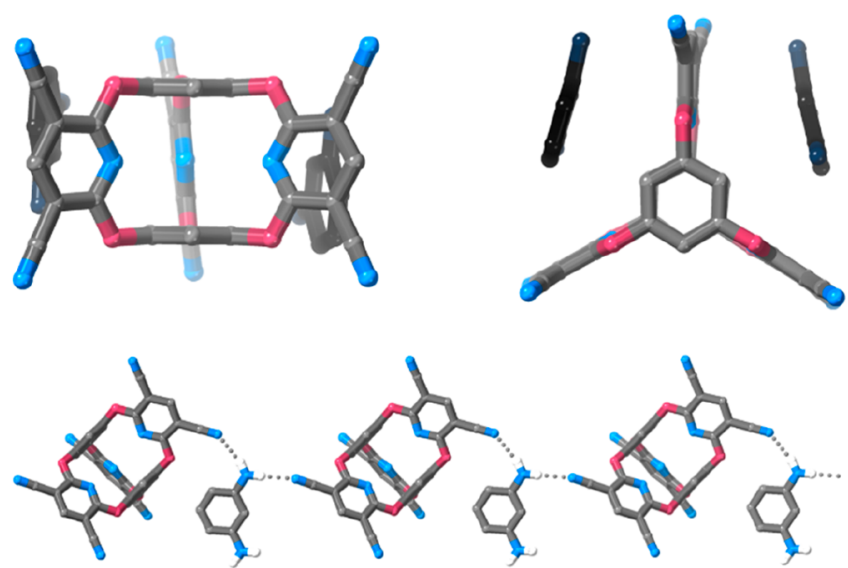

Figure 7 . Views of the structure of $1 \cdot(\mathrm{DAB})_{2}$ : views of asymmetric unit showing the similarity of structure to solvatomorphs of $\mathbf{1}$ (Figure 2), and a hydrogen bonded $1 \mathrm{D}$ chain formed from $\mathbf{1}$ and DAB (most hydrogen atoms omitted for clarity).

Table 1. Structures of Products Formed from Crystallizations of $1^{a}$

\begin{tabular}{|c|c|}
\hline guest & product \\
\hline no guest & $1^{\mathrm{Z0.5}}$ \\
\hline \multicolumn{2}{|l|}{ methanol } \\
\hline 6 equiv & $1^{\mathrm{Z0.5}}$ \\
\hline 25 equiv & $1^{\mathrm{Z0.5}}$ \\
\hline \multicolumn{2}{|l|}{ Res } \\
\hline 1 equiv & $1^{\mathrm{Z2}}$ \\
\hline 2 equiv & mainly $\mathbf{1}^{\mathrm{Z} 2}$, some amorphous powder ${ }^{b}$ \\
\hline 6 equiv & mainly $\mathbf{1}^{\mathrm{Z2}}$, some amorphous powder ${ }^{b}$ \\
\hline \multicolumn{2}{|l|}{ PG } \\
\hline 1 equiv & mainly $1^{\mathrm{Z} 3}$, a few crystals of $1^{\mathrm{Z} 2}$ \\
\hline 2 equiv & $1^{\mathrm{Z2}}$ \\
\hline 6 equiv & $1^{\mathrm{Z2}}$ \\
\hline \multicolumn{2}{|l|}{ HQ } \\
\hline 1 equiv & $1^{\mathrm{Z} 2}$ \\
\hline 2 equiv & mainly $\mathbf{1} \cdot(\mathbf{H Q})_{0.5}$, very few crystals of $\mathbf{1}^{\mathrm{Z} 2}$ \\
\hline 6 equiv & $1 \cdot(H Q)_{0.5}$ \\
\hline \multicolumn{2}{|l|}{$\mathbf{D A B}^{a}$} \\
\hline 1 equiv $^{a}$ & $1 \cdot(\mathrm{DAB})_{2}$ \\
\hline 2 equiv $^{a}$ & $1 \cdot(\mathrm{DAB})_{2}$ \\
\hline 6 equiv $^{a}$ & $1 \cdot(\mathrm{DAB})_{2}$ \\
\hline
\end{tabular}

${ }^{a}$ All crystallizations conducted by vapor diffusion of diethyl ether into a solution of $\mathbf{1}$ and guest in ethyl acetate, with the exception of DAB crystallizations: for solubility reasons, these were conducted by layering solutions of $\mathbf{1}$ and DAB in ethyl acetate. ${ }^{b}$ NMR spectroscopy suggests this powder is Res.

While this discussion and Table 1 focus on SCXRD studies, visual analysis of crystal habit, PXRD, and NMR spectroscopy (to determine whether or not cocrystals have formed) are all consistent with these results.

It is well-known that addition of modifiers can affect the rate and outcome of crystal growth: ${ }^{36,46,47}$ typically by affecting the rate of crystallization and favoring a slower- or faster-forming phase. While we cannot rule out this effect in our studies, it does not appear to have a dramatic role-crystals of all phases formed in 1-2 days (i.e., no phase crystallized particularly quickly or slowly).

The role of the guests also does not appear to be purely due to their hydrogen bonding ability: crystallization using either 6 or 25 equiv of methanol gave the same phase $\left(1^{\mathrm{zo.5}}\right)$ as when no guest was present. It is noteworthy that the $1^{\mathrm{Z0.5}}$ phase is not observed at all when any of Res, PG, HQ or DAB are present.

Nonetheless, solvent clearly has an important role: when $\mathbf{1}$ was crystallized in the presence of excess $\mathrm{HQ}$ from $\mathrm{MeCN} /$ $\mathrm{Et}_{2} \mathrm{O}$ rather than EtOAc/ $\mathrm{Et}_{2} \mathrm{O}$, we obtained $1 \cdot 2 \mathrm{MeCN}$. While a detailed study of the effect of guests and solvent together is beyond the scope of this investigation, it is notable that in the polar organic solvent $\mathrm{MeCN}$, a new phase is not observed. This may suggest that the role of the guests is to associate with the cage during crystal formation and direct crystallization to different forms and that this effect is negated by a polar solvent (which reduces interactions between cage and guest).

Despite obtaining six different structures of $\mathbf{1}$ without a guest, solvent is always present, occupying $17-30 \%$ of the unit cell volume. ${ }^{48}$ Indeed, the only solvent-free structure is that of $1 \cdot(\mathrm{DAB})_{2}$, where the DAB guests effectively take up the space that would otherwise be occupied by solvent molecules. Presumably, there is no easy way for $\mathbf{1}$ to pack efficiently, and so some void space is inevitable. Despite the varied structures formed, the volume $/ Z$ (i.e., volume per cage molecule and associated guests/solvents) does not change significantly, ranging from $855 \AA^{3}$ in the closest-packed structures (1. $2 \mathrm{MeCN}$ and $\mathbf{1}^{\mathrm{Z} 2}$ ) to $954 \AA^{3}$ in the least closely packed guestfree structure (1.2EtOAc). Guest cocrystallization results in a slight expansion of these values $\left[969 \AA^{3}\right.$ for $\mathbf{1} \cdot(\mathbf{D A B})_{2}$ and $1036 \AA^{3}$ in $\left.\mathbf{1} \cdot(\mathbf{H Q})_{0.5}\right]$.

Interestingly, the slightly larger volume/cage in the structure of $1 \cdot(H Q)_{0.5}$ results in moderately large channels running through the structure (Figure 6). While this structure is not particularly stable to drying, this does suggest that if stronger hydrogen bonding-accepting or -donating groups can be designed into intrinsically porous organic cage molecules, then it may be possible to use guests to order the pores present in these cages. Alternatively, an appropriate guest could be used to introduce extrinsic porosity.

\section{CONCLUSIONS}

We have prepared several different solvatomorphs of the organic cage molecule $\mathbf{1}$ and studied the effect of hydrogen bonding guests on crystal formation. Addition of Res, PG, HQ or DAB gave access to different crystal phases, which could not be isolated in the absence of these guests. In the case of HQ and DAB, we were able to isolate cocrystals of these guests and the cage where hydrogen bonds are present between some of the nitrile groups on the cage and the guests.

The cage used in this study is not intrinsically porous and does not contain accessible strong hydrogen bonding groups. However, we suggest this approach may offer a way to effectively order intrinsically porous cages (allowing for guest diffusion through ordered pores) or to introduce extrinsic pores. This would require cage molecules that are functionalized with strongly hydrogen bond-donating and/or -accepting groups, and work toward this goal is underway in our laboratories.

\section{EXPERIMENTAL SECTION}

Synthesis. Organic Synthesis. Cage compound 1 was prepared in one step from phloroglucinol and 2,6-dichloro-3,5-dicyanopyridine as described by Katz. ${ }^{32}$ Other chemicals and solvents were bought from commercial suppliers and used as received. 
Solvatomorph Crystal Growth. Crystals of 1-2EtOAc were prepared as described by Katz, ${ }^{32}$ by recrystallization from hot EtOAc. Crystals of $1^{\text {Z0.5 }}$ were prepared by vapor diffusion of $\mathrm{Et}_{2} \mathrm{O}$ into an EtOAc solution of $\mathbf{1}$. Crystals of $\mathbf{1}$-2acetone were prepared by vapor diffusion of $\mathrm{Et}_{2} \mathrm{O}$ into an acetone solution of $\mathbf{1}$. Crystals of $\mathbf{1}$. $2 \mathrm{MeCN}$ were prepared by vapor diffusion of $\mathrm{Et}_{2} \mathrm{O}$ into an acetonitrile solution of 1 .

Crystallization with Hydrogen Bonding Guests. A solution of 1 (13 mg, $0.020 \mathrm{mmol}$ ) in EtOAc ( $2 \mathrm{~mL}$ ) was added to solid HQ PG, or Res resulting in the formation of clear solutions. These solutions were subjected to $\mathrm{Et}_{2} \mathrm{O}$ vapor diffusion, resulting in the formation of crystals, or cocrystals in the case HQ. Adding a solution of $\mathbf{1}$ in EtOAc to DAB did not result in the formation of a clear solution, and so cocrystals of $\mathbf{1} \cdot(\mathrm{DAB})_{2}$ were prepared by layering EtOAc solutions of 1 and DAB. Yields of cocrystals were $95 \%$ in the case of $\mathbf{1} \cdot(\mathrm{HQ})_{0.5}$ and $65 \%$ for $\mathbf{1} \cdot(\mathrm{DAB})_{2}$.

Crystals and cocrystals formed were studied by ${ }^{1} \mathrm{H}$ NMR spectroscopy, SCXRD, PXRD, and visual analysis of crystal morphology. Details of crystallization experiments and characterization are provided in the Supporting Information.

X-ray Crystallography. Data were collected using mirrormonochromated Mo $\mathrm{K} \alpha$ or mirror-monochromated $\mathrm{Cu} \mathrm{K} \alpha$ radiation on an Agilent SuperNova diffractometer. Crystals were cooled to 150 $\mathrm{K}$ using an open-flow cooling device. Raw frame data (including data reduction, interframe scaling, unit cell refinement, and absorption corrections) were processed using CrysAlis PRO. ${ }^{49}$ Structures were solved using SIR92 $2^{50}$ or SUPERFLIP ${ }^{51}$ and refined using full-matrix least-squares on $F^{2}$ within the CRYSTALS suite. ${ }^{52} \mathrm{C}-\mathrm{H}$ hydrogen atoms were generally visible in the Fourier difference map and were initially refined with restraints on bond lengths and angles, after which the positions were used as the basis for a riding model. ${ }^{53} \mathrm{O}-\mathrm{H}$ and $\mathrm{N}-\mathrm{H}$ hydrogen atoms were visible in the Fourier difference map and were refined with restraints on $\mathrm{X}-\mathrm{H}$ bond lengths and $\mathrm{R}-\mathrm{X}-\mathrm{H}$ bond angles. A summary of crystallographic data is given in Table S2.

\section{ASSOCIATED CONTENT}

\section{S Supporting Information}

The Supporting Information is available free of charge on the ACS Publications website at DOI: 10.1021/acs.cgd.9b00565.

Details of effects of guests on crystal growth, characterization of cocrystals, solution phase binding studies, packing diagrams, additional crystallographic data (PDF)

\section{Accession Codes}

CCDC 1906148-1906154 contain the supplementary crystallographic data for this paper. These data can be obtained free of charge via www.ccdc.cam.ac.uk/data_request/cif, or by emailing data_request@ccdc.cam.ac.uk, or by contacting The Cambridge Crystallographic Data Centre, 12 Union Road, Cambridge CB2 1EZ, UK; fax: +44 1223336033.

\section{AUTHOR INFORMATION}

\section{Corresponding Author}

*E-mail: nicholas.white@anu.edu.au. Web: www.nwhitegroup. com.

\section{ORCID}

Nicholas G. White: 0000-0003-2975-0887

\section{Notes}

The authors declare no competing financial interest.

\section{ACKNOWLEDGMENTS}

We thank Prof. Christian Doonan (University of Adelaide) for helpful discussions.

\section{REFERENCES}

(1) Tozawa, T.; Jones, J. T. A.; Swamy, S. I.; Jiang, S.; Adams, D. J.; Shakespeare, S.; Clowes, R.; Bradshaw, D.; Hasell, T.; Chong, S. Y.; Tang, C.; Thompson, S.; Parker, J.; Trewin, A.; Bacsa, J.; Slawin, A. M. Z.; Steiner, A.; Cooper, A. I. Porous organic cages. Nat. Mater. 2009, 8, 973.

(2) Zhang, G.; Mastalerz, M. Organic cage compounds - from shapepersistency to function. Chem. Soc. Rev. 2014, 43, 1934-1947.

(3) Hasell, T.; Cooper, A. I. Porous organic cages: soluble, modular and molecular pores. Nat. Rev. Mater. 2016, 1, 16053.

(4) Tranchemontagne, D. J.; Ni, Z.; O’Keeffe, M.; Yaghi, O. M. Reticular Chemistry of Metal-Organic Polyhedra. Angew. Chem., Int. Ed. 2008, 47, 5136-5147.

(5) Yoshizawa, M.; Klosterman, J. K.; Fujita, M. Functional Molecular Flasks: New Properties and Reactions within Discrete, Self-Assembled Hosts. Angew. Chem., Int. Ed. 2009, 48, 3418-3438.

(6) Lu, Z.; Knobler, C. B.; Furukawa, H.; Wang, B.; Liu, G.; Yaghi, O. M. Synthesis and Structure of Chemically Stable Metal-Organic Polyhedra. J. Am. Chem. Soc. 2009, 131, 12532-12533.

(7) Duriska, M. B.; Neville, S. M.; Lu, J.; Iremonger, S. S.; Boas, J. F.; Kepert, C. J.; Batten, S. R. Systematic Metal Variation and Solvent and Hydrogen-Gas Storage in Supramolecular Nanoballs. Angew. Chem., Int. Ed. 2009, 48, 8919-8922.

(8) Cook, T. R.; Zheng, Y.-R.; Stang, P. J. Metal-Organic Frameworks and Self-Assembled Supramolecular Coordination Complexes: Comparing and Contrasting the Design, Synthesis, and Functionality of Metal-Organic Materials. Chem. Rev. 2013, 113, 734-777.

(9) Jin, Y.; Voss, B. A.; Jin, A.; Long, H.; Noble, R. D.; Zhang, W. Highly CO2-Selective Organic Molecular Cages: What Determines the CO2 Selectivity. J. Am. Chem. Soc. 2011, 133, 6650-6658.

(10) Jin, Y.; Voss, B. A.; McCaffrey, R.; Baggett, C. T.; Noble, R. D.; Zhang, W. Microwave-assisted syntheses of highly CO2-selective organic cage frameworks (OCFs). Chem. Sci. 2012, 3, 874-877.

(11) Li, J.-R.; Timmons, D. J.; Zhou, H.-C. Interconversion between Molecular Polyhedra and Metal-Organic Frameworks. J. Am. Chem. Soc. 2009, 131, 6368-6369.

(12) Ma, M.-L.; Li, X.-Y.; Wen, K. Coordination-Driven SelfAssembly of a Discrete Molecular Cage and an Infinite Chain of Coordination Cages Based on ortho-Linked Oxacalix[2]benzene[2]pyrazine and Oxacalix[2] arene[2]pyrazine. J. Am. Chem. Soc. 2009, $131,8338-8339$.

(13) Swamy, S. I.; Bacsa, J.; Jones, J. T. A.; Stylianou, K. C.; Steiner, A.; Ritchie, L. K.; Hasell, T.; Gould, J. A.; Laybourn, A.; Khimyak, Y. Z.; Adams, D. J.; Rosseinsky, M. J.; Cooper, A. I. A Metal-Organic Framework with a Covalently Prefabricated Porous Organic Linker. J. Am. Chem. Soc. 2010, 132, 12773-12775.

(14) Wang, H.-N.; Meng, X.; Yang, G.-S.; Wang, X.-L.; Shao, K.-Z.; Su, Z.-M.; Wang, C.-G. Stepwise assembly of metal-organic framework based on a metal-organic polyhedron precursor for drug delivery. Chem. Commun. 2011, 47, 7128-7130.

(15) Chen, L.; Chen, Q.; Wu, M.; Jiang, F.; Hong, M. Controllable Coordination-Driven Self-Assembly: From Discrete Metallocages to Infinite Cage-Based Frameworks. Acc. Chem. Res. 2015, 48, 201-210.

(16) Zhang, L.; Xiang, L.; Hang, C.; Liu, W.; Huang, W.; Pan, Y. From Discrete Molecular Cages to a Network of Cages Exhibiting Enhanced CO2 Adsorption Capacity. Angew. Chem., Int. Ed. 2017, 56, 7787-7791.

(17) Gosselin, E. J.; Lorzing, G. R.; Trump, B. A.; Brown, C. M.; Bloch, E. D. Gas adsorption in an isostructural series of pillared coordination cages. Chem. Commun. 2018, 54, 6392-6395.

(18) Cao, L.; Wang, P.; Miao, X.; Dong, Y.; Wang, H.; Duan, H.; Yu, Y.; Li, X.; Stang, P. J. Diamondoid Supramolecular Coordination Frameworks from Discrete Adamantanoid Platinum(II) Cages. J. Am. Chem. Soc. 2018, 140, 7005-7011.

(19) Zhang, C.; Wang, F.; Patil, R. S.; Barnes, C. L.; Li, T.; Atwood, J. L. Hierarchical Self-Assembly of Supramolecular Coordination Polymers Using Giant Metal-Organic Nanocapsules as Building Blocks. Chem. - Eur. J. 2018, 24, 14335-14340. 
(20) Dalton, H. L.; Lynes, A. D.; Twamley, B.; Byrne, K.; Schmitt, W.; Hawes, C. S.; Gunnlaugsson, T. Exploring the reversible hostguest chemistry of a crystalline octanuclear $\mathrm{Ag}(\mathrm{i})$ metallosupramolecular macrocycle formed from a simple pyrazinylpyridine ligand. Dalton Trans. 2018, 47, 17266-17275.

(21) Wang, D.-X.; Wang, Q.-Q.; Han, Y.; Wang, Y.; Huang, Z.-T.; Wang, M.-X. Versatile Anion $-\pi$ Interactions between Halides and a Conformationally Rigid Bis(tetraoxacalix[2]arene[2]triazine) Cage and Their Directing Effect on Molecular Assembly. Chem. - Eur. J. 2010, 16, 13053-13057.

(22) Yoon, M.; Suh, K.; Kim, H.; Kim, Y.; Selvapalam, N.; Kim, K. High and Highly Anisotropic Proton Conductivity in Organic Molecular Porous Materials. Angew. Chem., Int. Ed. 2011, 50, 7870-7873.

(23) Li, N.; Jiang, F.; Chen, L.; Li, X.; Chen, Q.; Hong, M. From discrete octahedral nanocages to $1 \mathrm{D}$ coordination polymer: Coordination-driven a single-crystal-to-single-crystal transformation via anion exchange. Chem. Commun. 2011, 47, 2327-2329.

(24) Liu, T.-F.; Chen, Y.-P.; Yakovenko, A. A.; Zhou, H.-C. Interconversion between Discrete and a Chain of Nanocages: SelfAssembly via a Solvent-Driven, Dimension-Augmentation Strategy. J. Am. Chem. Soc. 2012, 134, 17358-17361.

(25) Slater, A. G.; Little, M. A.; Pulido, A.; Chong, S. Y.; Holden, D.; Chen, L.; Morgan, C.; Wu, X.; Cheng, G.; Clowes, R.; Briggs, M. E.; Hasell, T.; Jelfs, K. E.; Day, G. M.; Cooper, A. I. Reticular synthesis of porous molecular 1D nanotubes and 3D networks. Nat. Chem. 2017, 9, 17.

(26) Liu, M.; Chen, L.; Lewis, S.; Chong, S. Y.; Little, M. A.; Hasell, T.; Aldous, I. M.; Brown, C. M.; Smith, M. W.; Morrison, C. A.; Hardwick, L. J.; Cooper, A. I. Three-dimensional protonic conductivity in porous organic cage solids. Nat. Commun. 2016, 7, 12750 .

(27) Ju, Z.; Liu, G.; Chen, Y.-S.; Yuan, D.; Chen, B. From Coordination Cages to a Stable Crystalline Porous Hydrogen-Bonded Framework. Chem. - Eur. J. 2017, 23, 4774-4777.

(28) Wang, X.-Y.; Zhu, J.; Wang, Q.-Q.; Ao, Y.-F.; Wang, D.-X. Anion- $\pi$-Directed Self-Assembly between Di- and Trisulfonates and a Rigid Molecular Cage with Three Electron-Deficient V-Clefts. Inorg. Chem. 2019, 58, 5980-5987.

(29) Luo, J.; Wang, J.-W.; Zhang, J.-H.; Lai, S.; Zhong, D.-C. Hydrogen-bonded organic frameworks: design, structures and potential applications. CrystEngComm 2018, 20, 5884-5898.

(30) Lin, R.-B.; He, Y.; Li, P.; Wang, H.; Zhou, W.; Chen, B. Multifunctional porous hydrogen-bonded organic framework materials. Chem. Soc. Rev. 2019, 48, 1362-1389.

(31) Hisaki, I.; Xin, C.; Takahashi, K.; Nakamura, T. Designing Hydrogen-Bonded Organic Frameworks (HOFs) with Permanent Porosity. Angew. Chem., Int. Ed. 2019, DOI: 10.1002/anie.201902147.

(32) Katz, J. L.; Selby, K. J.; Conry, R. R. Single-Step Synthesis of $D_{3 \mathrm{~h}}$-Symmetric Bicyclooxacalixarenes. Org. Lett. 2005, 7, 3505-3507.

(33) Le Questel, J.-Y.; Berthelot, M.; Laurence, C. Hydrogen-bond acceptor properties of nitriles: a combined crystallographic and $a b$ initio theoretical investigation. J. Phys. Org. Chem. 2000, 13, 347-358.

(34) Steiner, T. The Hydrogen Bond in the Solid State. Angew. Chem., Int. Ed. 2002, 41, 48-76.

(35) While neutral nitrile groups are relatively weak hydrogen bond acceptors, nitrile-containing anions have been shown to form frameworks assembled through relatively short hydrogen bonds: Turner, D. R.; Edwards, A. J.; Piltz, R. O. CrystEngComm 2012, 14, 6447-6451.

(36) Jones, F.; Ogden, M. I. Controlling crystal growth with modifiers. CrystEngComm 2010, 12, 1016-1023.

(37) Throughout this work, we define a cocrystal as a crystalline material composed of two different molecular compounds, neither of which are solvent. See refs 38 and 39 for a more detailed discussion.

(38) Aitipamula, S.; Banerjee, R.; Bansal, A. K.; Biradha, K.; Cheney, M. L.; Choudhury, A. R.; Desiraju, G. R.; Dikundwar, A. G.; Dubey, R.; Duggirala, N.; Ghogale, P. P.; Ghosh, S.; Goswami, P. K.; Goud, N. R.; Jetti, R. R. K. R.; Karpinski, P.; Kaushik, P.; Kumar, D.; Kumar,
V.; Moulton, B.; Mukherjee, A.; Mukherjee, G.; Myerson, A. S.; Puri, V.; Ramanan, A.; Rajamannar, T.; Reddy, C. M.; Rodriguez-Hornedo, N.; Rogers, R. D.; Row, T. N. G.; Sanphui, P.; Shan, N.; Shete, G.; Singh, A.; Sun, C. C.; Swift, J. A.; Thaimattam, R.; Thakur, T. S.; Kumar Thaper, R.; Thomas, S. P.; Tothadi, S.; Vangala, V. R.; Variankaval, N.; Vishweshwar, P.; Weyna, D. R.; Zaworotko, M. J. Polymorphs, Salts, and Cocrystals: What's in a Name? Cryst. Growth Des. 2012, 12, 2147-2152.

(39) Kersten, K.; Kaur, R.; Matzger, A. Survey and analysis of crystal polymorphism in organic structures. IUCrJ 2018, 5, 124-129.

(40) For a recent detailed study of solvatomorphism in Reichardt's dye, see: Pike, S. J.; Bond, A. D.; Hunter, C. A. Solvatomorphism of Reichardt's dye. CrystEngComm 2018, 20, 2912-2915.

(41) Spek, A. L. PLATON SQUEEZE: a tool for the calculation of the disordered solvent contribution to the calculated structure factors. Acta Crystallogr., Sect. C: Struct. Chem. 2015, C71, 9-18.

(42) Alvarez, S. A cartography of the van der Waals territories. Dalton Trans. 2013, 42, 8617-8636.

(43) Calculated using Mercury ${ }^{44}$ with a probe radius of $1.2 \AA$. It should be noted that the solvent-accessible volume is much smaller ( $7 \%$ of the unit cell volume using a probe radius of $1.82 \AA$ ). See the Supporting Information for a more detailed analysis.

(44) Macrae, C. F.; Bruno, I. J.; Chisholm, J. A.; Edgington, P. R.; McCabe, P.; Pidcock, E.; Rodriguez-Monge, L.; Taylor, R.; van de Streek, J.; Wood, P. A. Mercury CSD 2.0 - new features for the visualization and investigation of crystal structures. J. Appl. Crystallogr. 2008, 41, 466-470.

(45) It was also possible to isolate crystals of this form when 0.5 equiv of $\mathrm{HQ}$ was present during crystallization, although in this case a mixture of crystals appears to form.

(46) Zahn, G.; Zerner, P.; Lippke, J.; Kempf, F. L.; Lilienthal, S.; Schröder, C. A.; Schneider, A. M.; Behrens, P. Insight into the mechanism of modulated syntheses: in situ synchrotron diffraction studies on the formation of $\mathrm{Zr}$-fumarate MOF. CrystEngComm 2014, 16, 9198-9207.

(47) McGuire, C. V.; Forgan, R. S. The surface chemistry of metalorganic frameworks. Chem. Commun. 2015, 51, 5199-5217.

(48) In some cases, voids filled with diffuse electron density that appear to correspond to disordered solvent were present. In these cases, PLATON-SQUEEZE ${ }^{41}$ was used to include the electron density in the model; see the discussion of individual structures for more details.

(49) CrysAlis PRO; Oxford Diffraction, 2011.

(50) Altomare, A.; Cascarano, G.; Giacovazzo, C.; Guagliardi, A.; Burla, M. C.; Polidori, G.; Camalli, M. SIR92 - a program for automatic solution of crystal structures by direct methods. J. Appl. Crystallogr. 1994, 27, 435.

(51) Palatinus, L.; Chapuis, G. SUPERFLIP. A computer program for the solution of crystal structures by charge flipping in arbitrary dimensions. J. Appl. Crystallogr. 2007, 40, 786-790.

(52) Betteridge, P. W.; Carruthers, J. R.; Cooper, R. I.; Prout, K.; Watkin, D. J. CRYSTALS version 12: software for guided crystal structure analysis. J. Appl. Crystallogr. 2003, 36, 1487.

(53) Cooper, R. I.; Thompson, A. L.; Watkin, D. J. CRYSTALS enhancements: dealing with hydrogen atoms in refinement. J. Appl. Crystallogr. 2010, 43, 1100-1107. 